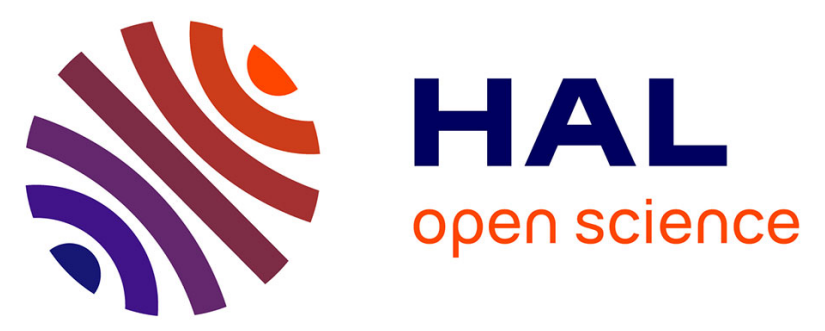

\title{
Séparation et reconstruction des ondes dans les barres élastiques et viscoélastiques à partir de mesures redondantes
}

Ramzi Othman, Marie-Noëlle Bussac, Pierre Collet, Gérard Gary

\section{- To cite this version:}

Ramzi Othman, Marie-Noëlle Bussac, Pierre Collet, Gérard Gary. Séparation et reconstruction des ondes dans les barres élastiques et viscoélastiques à partir de mesures redondantes. Comptes Rendus Mécanique, 2001, 329 (5), pp.369 - 376. 10.1016/S1620-7742(01)01343-5 . hal-01007282

\section{HAL Id: hal-01007282 \\ https://hal.science/hal-01007282}

Submitted on 9 Oct 2016

HAL is a multi-disciplinary open access archive for the deposit and dissemination of scientific research documents, whether they are published or not. The documents may come from teaching and research institutions in France or abroad, or from public or private research centers.
L'archive ouverte pluridisciplinaire HAL, est destinée au dépôt et à la diffusion de documents scientifiques de niveau recherche, publiés ou non, émanant des établissements d'enseignement et de recherche français ou étrangers, des laboratoires publics ou privés.

\section{(c)(1)}

Distributed under a Creative Commons Attribution| 4.0 International License 


\title{
Séparation et reconstruction des ondes dans les barres élastiques et viscoélastiques à partir de mesures redondantes
}

\author{
Ramzi OTHMAN a ${ }^{\text {, Marie-Noëlle BUSSAC }}{ }^{\text {b }}$, Pierre COLLET ${ }^{\text {b }}$, Gérard GARY ${ }^{\text {a }}$ \\ ${ }^{\text {a }}$ Laboratoire de mécanique des solides, UMR 7649, École polytechnique, 91128 Palaiseau, France \\ ${ }^{\text {b }}$ Centre de physique théorique, UMR 7644, École polytechnique, 91128 Palaiseau, France \\ Courriel : othman@Ims.polytechnique.fr
}

Résumé. La durée du chargement habituellement utilisée avec le système des barres de Hopkinson (ou SHPB pour Split Hopkinson Pressure Bar) est limitée par la nécessité de séparer les ondes se propageant dans des sens opposés. En utilisant le principe du maximum de vraisemblance, et à partir de $N$ mesures de déformation et $P$ mesures de vitesse matérielle $(N+P \geqslant 2)$, nous construisons une nouvelle méthode de séparation des ondes dans les barres. Cette méthode permet de calculer, sans limitation de durée, les contraintes, les déformations, les déplacements et les vitesses matérielles en tout point de la barre. Elle tient compte des effets dispersifs aussi bien dans les barres élastiques que dans les barres viscoélastiques. Elle est illustrée sur une barre libre pour laquelle le déplacement mesuré est 30 fois supérieur à celui qui serait atteint par la méthode classique. @ 2001 Académie des sciences/Éditions scientifiques et médicales Elsevier SAS

ondes / séparation des ondes / barres de Hopkinson / barres de Kolsky / essais dynamiques

\section{Separation and rebuilding of waves in elastic and viscoelastic bars using redundant measures}

Abstract. When using a classical SHPB (Split Hopkinson Pressure Bar) set-up, the useful measuring time is limited by the need of separating the waves propagating in opposite directions. A new method for separating and rebuilding waves in finite bars was developed, with no time limit, from $N$ strain measurements and $P$ particle velocity measurements $(N+P \geqslant 2)$. It takes account of the wave dispersion in elastic and viscoelastic bars. The method is illustrated with the loading of a free bar where the measured displacement is 30 times longer than the displacement that would be obtained with the classical method. $\odot 2001$ Académie des sciences/Éditions scientifiques et médicales Elsevier SAS

waves / wave separation / Hopkinson bar / Kolsky bar / dynamic tests

\section{Abridged English version}

The loading time (and then the useful measuring time) in the SHPB system is limited because the analysis of the measurements requires the separation of waves moving in opposite directions [1,2]. Some solutions for separating these waves without time limits are already available. However, they assume a 
one-dimensional propagation model [3,5] or they are iterative [6,7] or they are mixed between time and frequency domain [8]. They all are based on a two-point measurement method.

In this paper a new method based on exact calculations in the frequency domain is developed. It requires $N$ strains and $P$ velocities measurements $(N+P \geqslant 2)$ made on an elastic or viscoelastic bar. We assume that each measurement provides the actual strain (or velocity) to which is added a white noise. These white noises are supposed to be statistically independent and are adjusted so that they have the same variance. Using the Maximum Likelihood Principle, the waves propagating in opposite directions are expressed as functions of the measured signals and the bar dispersion relation (equations (9.a) and (9.b)). An experimental application with an aluminium bar showed that a 'three-strain-one-velocity' measurement provides a solution giving satisfactory results (figure 8). A two-strain solution amplifies the noise in the stress computation. It accumulates errors due to imprecise zero-electric measurements in the displacement computation (figures 4 and 5). A three-strain solution stabilises the noise as verified in [17] but the displacement computed is still not satisfactory (figures 6 and 7). The good results obtained with the 'threestrain-one-velocity solution' allow for a significant increase in the observation time. It makes possible to obtain precise measurements up to large strains even at medium strain rates, in a test range in between that of mechanical testing machines and that of Hopkinson bars.

\section{Introduction}

Les barres de Hopkinson (ou barres de Kolsky, ou SHPB pour Split Hopkinson Pressure Bar) sont un moyen d'essai couramment utilisé en dynamique qui est bien adapté à la mesure du comportement des matériaux. Cette technique doit sa configuration actuelle à Kolsky [1] faisant suite aux travaux de pionnier de Hopkinson [2]. Dans cette configuration, la durée du chargement est limitée par la nécessité de séparer les ondes se propageant dans des sens opposés. La séparation y est en effet réalisée en dimensionnant le système de sorte que les ondes ne se superposent pas aux points de mesure.

Dans les configurations où les ondes se superposent, le problème de leur séparation a fait l'objet d'études fondées sur des mesures en deux points. Une solution utilisant une méthode itérative a été proposée dans le cadre de la propagation non dispersive des ondes [3-5]. Cette méthode a été étendue par Zhao et Gary [6,7] en prenant en compte de manière approchée la dispersion et l'amortissement dans les barres élastiques et viscoélastiques. Bacon [8], a proposé un algorithme de résolution dans l'espace fréquentiel d'une formule exacte proposée dans [7].

Dans cet article, nous présentons une méthode de séparation des ondes utilisant des mesures redondantes de déformation et (ou) de vitesse matérielle (qu'on désignera simplement par «vitesse» dans la suite), dont le nombre peut alors dépasser deux. La méthode, qui résout les difficultés mentionnées dans les études précédentes, est validée expérimentalement sur une barre d'aluminium.

\section{Propagation des ondes dans les barres élastiques et viscoélastiques}

Nous considérons dans la suite une barre de longueur $L$ (figure 1 ) supposée viscoélastique de module de Young complexe $E^{*}(\omega)$. Le cas élastique correspond à $E^{*}(\omega)=E_{0}$ (où $E_{0}$ est une constante réelle). Les déplacements et les déformations sont supposés homogènes dans une section de la barre repérée par son abscisse $x$. En outre, seul le premier mode de propagation des ondes longitudinales est supposé excité par l'impact du projectile [9]. Suivant [10] et [11], l'expression temporelle de la déformation s'écrit :

$$
\varepsilon(x, t)=\frac{1}{2 \pi} \int_{-\infty}^{+\infty} \tilde{\varepsilon}(x, \omega) \mathrm{e}^{\mathrm{i} \omega t} \mathrm{~d} \omega
$$


avec

$$
\tilde{\varepsilon}(x, \omega)=A(\omega) \mathrm{e}^{-\mathrm{i} \xi(\omega) x}+B(\omega) \mathrm{e}^{\mathrm{i} \xi(\omega) x}
$$

où le nombre d'onde complexe $\xi(\omega)$ s'écrit :

$$
\xi(\omega)=k(\omega)+\mathrm{i} \alpha(\omega)=\frac{\omega}{c(\omega)}+\mathrm{i} \alpha(\omega)
$$

$A(\omega)$ et $B(\omega)$ sont les composantes fréquentielles de la déformation des ondes montante et descendante à l'origine qu'on se propose de déterminer, $c(\omega)$ est la vitesse de phase des ondes longitudinales et $\alpha(\omega)$ le coefficient d'amortissement. La relation de dispersion $\xi(\omega)$ dépend de manière couplée de la géométrie de la barre et du matériau qui la constitue. Pour une barre de section circulaire, $\xi(\omega)$ est solution de l'équation de Pochhammer-Chree [12-14]. On déduit de (2) que les composantes fréquentielles de la contrainte, de la vitesse et du déplacement sont données respectivement par :

$$
\begin{aligned}
& \tilde{\sigma}(x, \omega)=E^{*}(\omega)\left(A(\omega) \mathrm{e}^{-\mathrm{i} \xi(\omega) x}+B(\omega) \mathrm{e}^{\mathrm{i} \xi(\omega) x}\right) \\
& \tilde{v}(x, \omega)=-\omega \frac{A(\omega) \mathrm{e}^{-\mathrm{i} \xi(\omega) x}-B(\omega) \mathrm{e}^{\mathrm{i} \xi(\omega) x}}{\xi(\omega)} \\
& \tilde{u}(x, \omega)=\mathrm{i} \frac{A(\omega) \mathrm{e}^{-\mathrm{i} \xi(\omega) x}-B(\omega) \mathrm{e}^{\mathrm{i} \xi(\omega) x}}{\xi(\omega)}
\end{aligned}
$$

\section{Séparation des ondes}

Les formules (4) montrent que connaissant $\xi(\omega), E^{*}(\omega), A(\omega)$ et $B(\omega)$, la déformation, la contrainte, le déplacement et la vitesse matérielle sont déterminés en toute section de la barre. Dans ce qui suit $\xi(\omega)$ et $E^{*}(\omega)$ sont supposés connus. Nous nous proposons de déterminer les ondes montante et descendante caractérisées par $A(\omega)$ et $B(\omega)$ à partir de $N$ mesures de déformation et $P$ mesures de vitesse effectuées aux sections d'abscisse $x_{j}, j=1, \ldots, N$ (respectivement $x_{k+N}, k=1, \ldots, P$ ) et notées $\hat{\varepsilon}_{j}(t), j=1, \ldots, N$ (respectivement $\hat{v}_{k}(t), k=1, \ldots, P$ ). Nous supposons que ces mesures correspondent à la valeur exacte de la déformation augmentée d'une erreur que nous modélisons par des bruits blancs indépendants $b_{r}(t)$, $r=1, \ldots, N+P$, d'amplitude $1 / w_{r}$ associés à chaque mesure.

$$
\begin{aligned}
\hat{\varepsilon}_{j}(t) & =\varepsilon\left(x_{j}, t\right)+b_{j}(t), \quad j=1, \ldots, N \\
\text { et } \quad \hat{v}_{k}(t) & =v\left(x_{k+N}, \omega\right)+b_{k+N}(t), \quad k=1, \ldots, P
\end{aligned}
$$

L'application du principe du maximum de vraisemblance consiste à déterminer les fonctions $A(\omega)$ et $B(\omega)$ qui minimisent la fonction réelle de vraisemblance définie par la relation (6) [15,11] :

$$
F=\int_{-\infty}^{+\infty}\left\{\sum_{j=1}^{N} w_{j}^{2}\left(\hat{\varepsilon}_{j}(t)-\varepsilon\left(x_{j}, t\right)\right)^{2}+\sum_{k=1}^{P} w_{k+N}^{2}\left(\hat{v}_{k}(t)-v\left(x_{k+N}, t\right)\right)^{2}\right\} \mathrm{d} t
$$

Nous retrouvons, comme attendu avec des bruits Gaussiens indépendants, l'expression de la fonctionnelle des moindres carrés. Compte tenu de (3) il vient, en utilisant la transformée de Fourier :

$$
\begin{aligned}
F= & \frac{1}{2 \pi} \int \sum_{j=1}^{N} w_{j}^{2}\left|\tilde{\hat{\varepsilon}}_{j}(\omega)-A(\omega) \mathrm{e}^{-\mathrm{i} \xi(\omega) x_{j}}-B(\omega) \mathrm{e}^{\mathrm{i} \xi(\omega) x_{j}}\right|^{2} \mathrm{~d} \omega \\
& +\frac{1}{2 \pi} \int \sum_{k=1}^{P} w_{k+N}^{2}\left|\tilde{\hat{v}}_{k}(\omega)+c^{*}(\omega) A(\omega) \mathrm{e}^{-\mathrm{i} \xi(\omega) x_{k+N}}-c^{*}(\omega) B(\omega) \mathrm{e}^{\mathrm{i} \xi(\omega) x_{k+N}}\right|^{2} \mathrm{~d} \omega
\end{aligned}
$$

où $c^{*}(\omega)$ dénote $\omega / \xi(\omega)$. 
Introduisons les notations suivantes, où $\bar{z}$ dénote le complexe conjugué de $z$ :

$$
\begin{aligned}
& h_{1}(\omega)=\sum_{j=1}^{N} w_{j}^{2} \mathrm{e}^{-\mathrm{i}(\xi(\omega)-\overline{\xi(\omega)}) x_{j}}+\left|c^{*}(\omega)\right|^{2} \sum_{k=1}^{P} w_{k+N}^{2} \mathrm{e}^{-\mathrm{i}(\xi(\omega)-\overline{\xi(\omega)}) x_{k+N}} \\
& h_{2}(\omega)=\sum_{j=1}^{N} w_{j}^{2} \mathrm{e}^{\mathrm{i}(\xi(\omega)-\overline{\xi(\omega)}) x_{j}}+\left|c^{*}(\omega)\right|^{2} \sum_{k=1}^{P} w_{k+N}^{2} \mathrm{e}^{\mathrm{i}(\xi(\omega)-\overline{\xi(\omega)}) x_{k+N}} \\
& g(\omega)=\sum_{j=1}^{N} w_{j}^{2} \mathrm{e}^{\mathrm{i}(\xi(\omega)+\overline{\xi(\omega)}) x_{j}}-\left|c^{*}(\omega)\right|^{2} \sum_{k=1}^{P} w_{k+N}^{2} \mathrm{e}^{\mathrm{i}(\xi(\omega)+\overline{\xi(\omega)}) x_{k+N}} \\
& E_{1}(\omega)=\sum_{j=1}^{N} w_{j}^{2} \mathrm{e}^{\mathrm{i} \overline{\xi(\omega)} x_{j}} \tilde{\hat{\varepsilon}}_{j}(\omega)-\sum_{k=1}^{P} w_{k+N}^{2} \mathrm{e}^{\mathrm{i} \overline{\xi(\omega)} x_{k+N}} c^{*}(\omega) \tilde{\hat{v}}_{k}(\omega) \\
& E_{2}(\omega)=\sum_{j=1}^{N} w_{j}^{2} \mathrm{e}^{-\mathrm{i} \overline{\xi(\omega)} x_{j}} \tilde{\hat{\varepsilon}}_{j}(\omega)+\sum_{k=1}^{P} w_{k+N}^{2} \mathrm{e}^{-\mathrm{i} \overline{\xi(\omega)} x_{k+N}} c^{*}(\omega) \tilde{\hat{v}}_{k}(\omega)
\end{aligned}
$$

La fonction $F$ s'écrit alors :

$$
F=\frac{1}{2 \pi} \int \mathrm{d} \omega\left[\langle\mathbf{X}(\omega) \mid T(\omega) \mathbf{X}(\omega)\rangle-\langle\mathbf{E}(\omega) \mid \mathbf{X}(\omega)\rangle-\langle\mathbf{X}(\omega) \mid \mathbf{E}(\omega)\rangle+\sum_{j=1}^{N} \overline{\tilde{\hat{\varepsilon}}_{j}(\omega)} \tilde{\hat{\varepsilon}}_{j}(\omega)\right]
$$

avec :

$$
\begin{aligned}
& \mathbf{X}(\omega)=\left(\begin{array}{l}
A(\omega) \\
B(\omega)
\end{array}\right), \quad \mathbf{E}(\omega)=\left(\begin{array}{l}
E_{1}(\omega) \\
E_{2}(\omega)
\end{array}\right) \\
& \text { et } T(\omega)=\left(\begin{array}{lc}
\frac{h_{1}(\omega)}{g(\omega)} & g(\omega) \\
h_{2}(\omega)
\end{array}\right)
\end{aligned}
$$

Le minimum de la fonction $F$ est atteint lorsque $\mathbf{X}(\omega)=T(\omega)^{-1} \mathbf{E}(\omega)$, soit :

$$
\begin{aligned}
& A(\omega)=\frac{h_{2}(\omega) E_{1}(\omega)-g(\omega) E_{2}(\omega)}{h_{1}(\omega) h_{2}(\omega)-g(\omega) \overline{g(\omega)}} \\
& \text { et } B(\omega)=\frac{h_{1}(\omega) E_{2}(\omega)-\overline{g(\omega)} E_{1}(\omega)}{h_{1}(\omega) h_{2}(\omega)-g(\omega) \overline{g(\omega)}}
\end{aligned}
$$

Lorsque $N=2$, le résultat correspond à l'inversion exacte de la relation (4.a) pour chaque jauge [7,8, 11]. Le dénominateur des expressions de $A(\omega)$ et $B(\omega)$ s'annule pour plusieurs valeurs réelles de $\omega[7,8]$. Les résonances correspondantes sont obtenues pour les composantes fréquentielles multiples de la célérité divisée par le double de la distance entre les jauges, comme cela est expliqué en détail dans [8]. Pour déterminer les expressions temporelles, nous contournons cette difficulté en inversant les transformées de Fourier sur une droite parallèle à la droite réelle. Pour assurer la causalité, celle-ci doit être située dans le demi-plan inférieur du plan complexe. En supposant la fonction $\xi(\omega)$ analytique, l'intégration ne dépend pas du chemin suivi $[16,11]$. Cette procédure est équivalente à l'application de la transformée de Laplace.

Elle est détaillée sur le diagramme ci-dessous, 


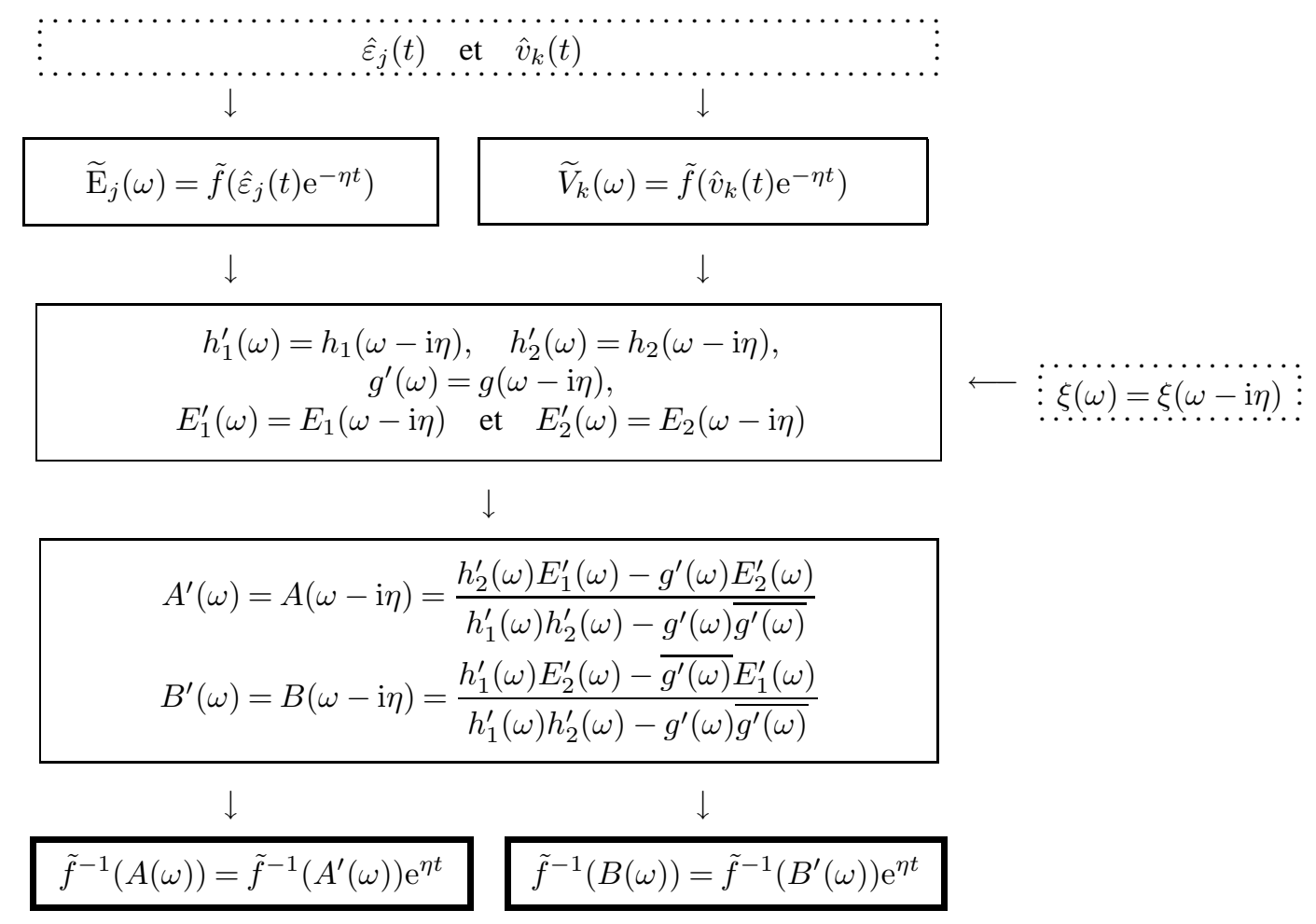

où $\tilde{f}$ et $\tilde{f}^{-1}$ dénotent respectivement la transformée de Fourier et la transformée de Fourier inverse. $\eta$ une constante positive.

\section{Validation expérimentale - Application à une barre d'aluminium}

\subsection{Description}

Nous considérons une barre d'aluminium de longueur $L=3,019 \mathrm{~m}$ et de diamètre $d=40 \mathrm{~mm}$ (figure 1 ). La masse volumique de la barre est de $2808 \mathrm{~kg} / \mathrm{m}^{3}$, le coefficient de poisson est $\nu=0,34$ et le module d'Young est $E_{0}=72,6 \mathrm{GPa}$. La relation de dispersion est alors déterminée en résolvant l'équation de Pochhammer-Chree [12-14] avec ces valeurs. Les mesures de déformations sont effectuées en trois points d'abscisses $x_{1}=0,345 \mathrm{~m}, x_{2}=1,514 \mathrm{~m}$ et $x_{3}=2,507 \mathrm{~m}$. En utilisant deux extensomètres optiques (type «TSI Zimmer PM100H») deux mesures de déplacement sont effectuées aux point d'abscisse $x_{4}=2,408 \mathrm{~m}$ et à l'extrémité libre de la barre $\left(x_{5}=L=3,019 \mathrm{~m}\right.$ - voir figure 1$)$. Un impacteur de longueur $l=1,2 \mathrm{~m}$ est projeté sur l'extrémité gauche de la barre à une vitesse $v_{\text {imp }}=5,78 \mathrm{~m} / \mathrm{s}$. Les mesures sont enregistrées à une fréquence d'échantillonnage $f_{\mathrm{e}}=500 \mathrm{kHz}$. On effectue la transformée de Fourier sans fenêtrage et sur 65536 points (figures 2 et 3 ).

\subsection{Reconstruction des ondes}

\subsubsection{Deux mesures de déformation}

Dans un premier temps, nous ne considérons que les déformations mesurées en $x_{1}$ et $x_{3}$. Nous vérifions que les bruits attachés à ces mesures sont des bruits blancs de même variance. Leurs poids seront donc pris égaux à $w_{1}=w_{3}=1$. Nous calculons les contraintes aux extrémités de la barre (figure 4 ) et le déplacement à l'extrémité libre (figure 5) en appliquant les résultats du $\S 3$ pour $N=2$ et $P=0$. Nous observons que le 


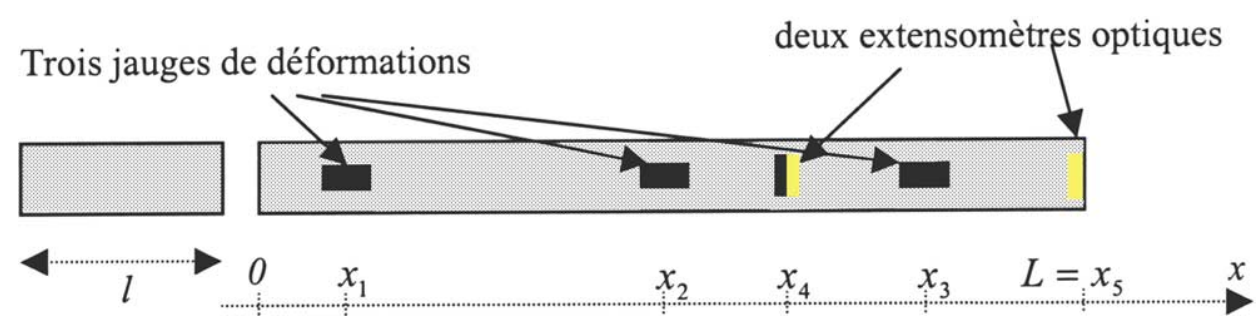

Figure 1. Dispositif expérimental.

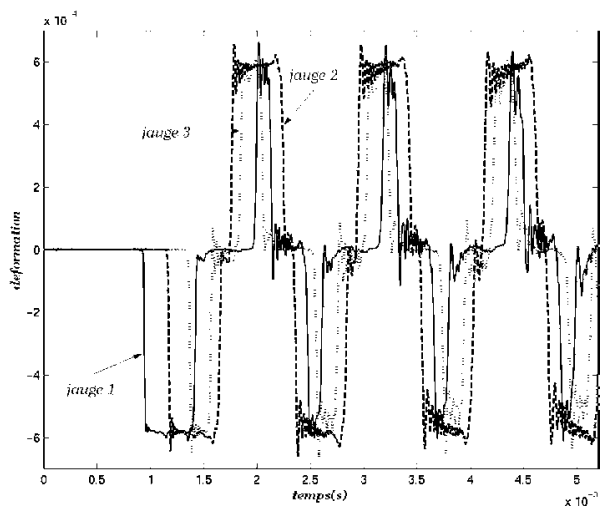

Figure 2. Déformations mesurées (pour la clarté de la figure, elles ne sont représentées que pour une durée de $5 \mathrm{~ms}$ ).

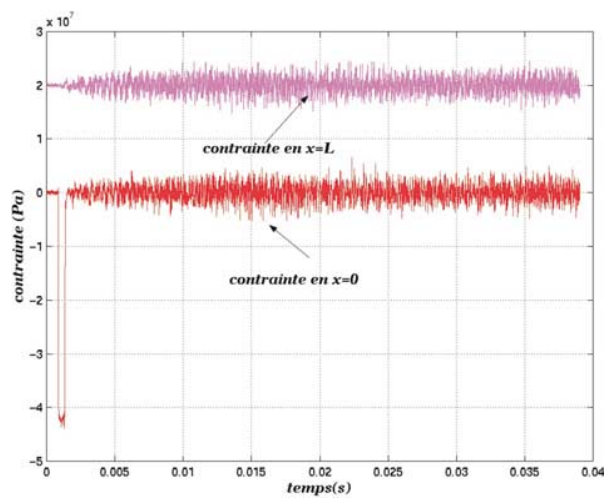

Figure 4. Contraintes aux deux extrémités reconstruites à partir de deux mesures de déformations (pour la clarté de la figure, la contrainte au bout libre est décalé de $20 \mathrm{MPa}$ ).
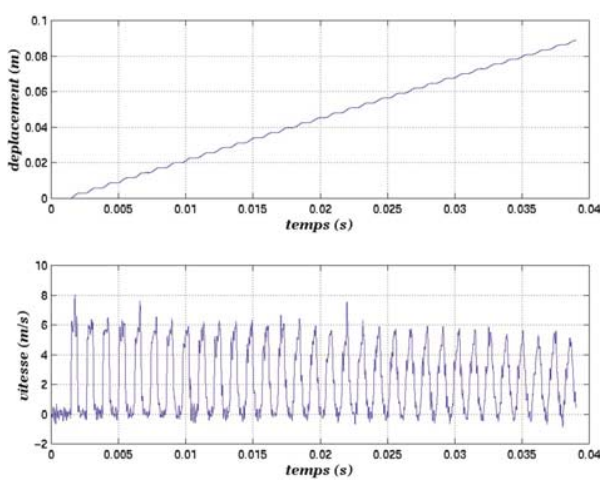

Figure 3. Déplacement mesuré en $x_{4}$ et vitesse correspondante calculée par dérivation.

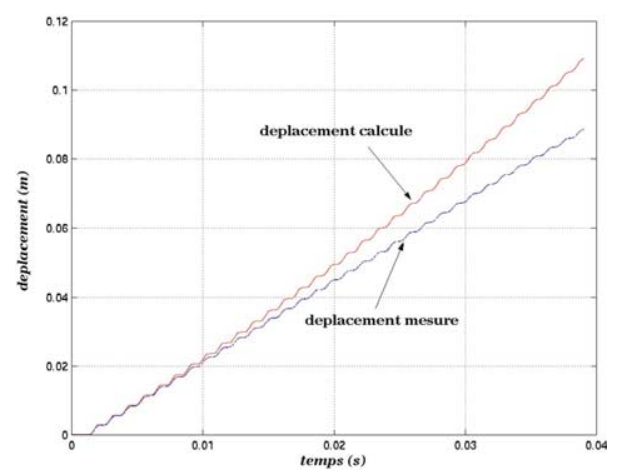

Figure 5. Comparaison des déplacements au bout libre mesuré par l'extensomètre optique et calculé à partir de deux mesures de déformation.

bruit est amplifié pour le calcul des contraintes (ou des déformations). De plus, le calcul des déplacements (ou des vitesses) diverge. On montre dans [11] que ce dernier effet est la conséquence d'une imparfaite mesure du zéro électrique théoriquement associé à une déformation nulle. L'erreur sur les contraintes est de l'ordre de $12 \%$ et l'erreur maximale sur le déplacement de $22 \%$. 


\subsubsection{Trois mesures de déformation}

Pour réduire l'effet d'amplification du bruit on introduit une troisième mesure redondante [17,11]. Dans ce cas $N=3, P=0$ et $w_{1}=w_{2}=w_{3}=1$. Les bruits sur les mesures ne sont plus amplifiés pour le calcul des contraintes (figure 6). L'erreur sur celles-ci est inférieure à $3 \%$ de l'amplitude maximum du signal : la méthode du maximum de vraisemblance révèle toute sa puissance. Néanmoins, l'introduction de la troisième mesure ne corrige pas complètement la dérive du déplacement : l'erreur maximale reste de l'ordre de $8 \%$ (figure 7 ).

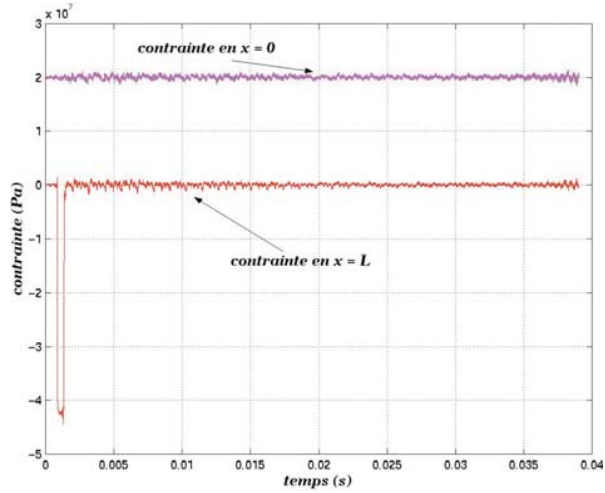

Figure 6. Contraintes aux deux bouts reconstruites avec trois mesures de déformations (pour la clarté de la figure la contrainte au bout libre est décalée de $20 \mathrm{MPa}$ ).

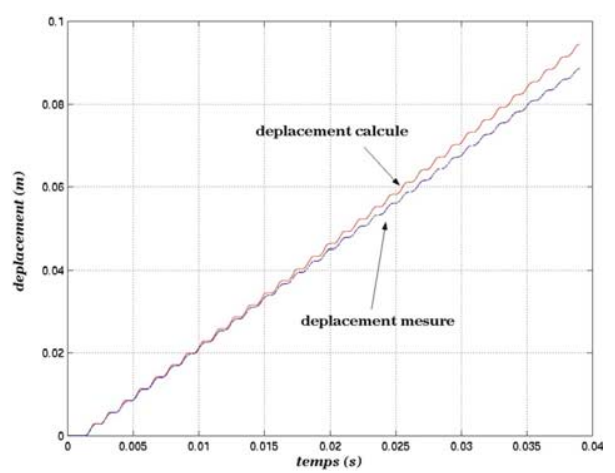

Figure 7. Déplacement au bout libre (calculé avec trois mesures de déformation) comparé au déplacement mesuré.
Figure 8. Déplacement au bout libre (calculé avec trois mesures de déformation et une mesure de vitesse) comparé au déplacement mesuré.

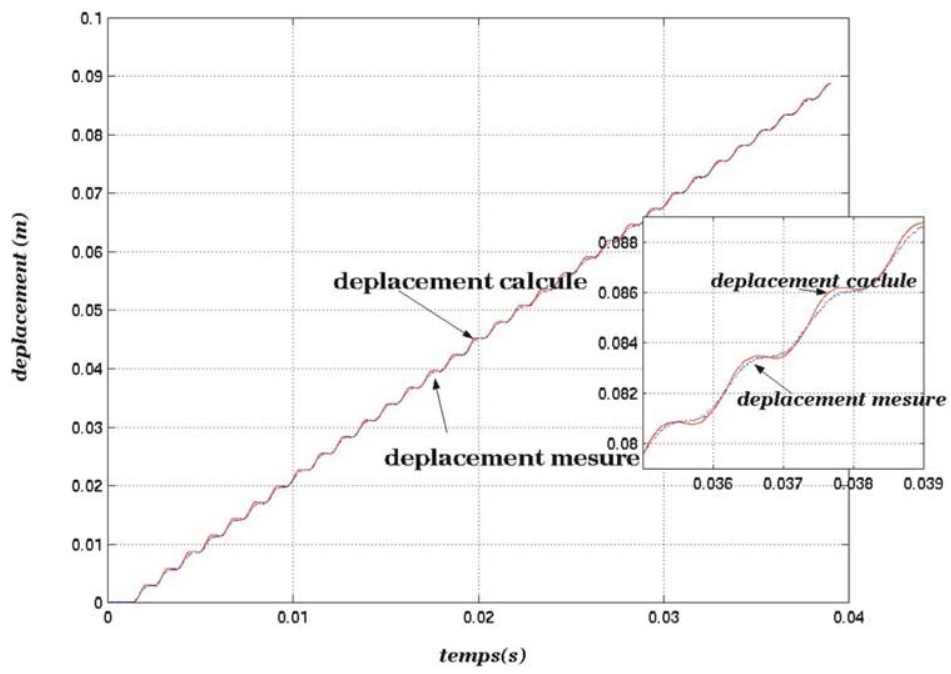

\subsubsection{Trois mesures de déformation et une mesure de vitesse}

Pour résoudre le problème, nous introduisons comme mesure supplémentaire celle de la vitesse (calculée en dérivant par rapport au temps le déplacement mesuré en $x_{4}$ ). Dans ce cas, $N=3, P=1$. Les mesures de vitesses et les mesures de déformations étant de même qualité nous prenons $w_{1}=w_{2}=w_{3}=1$ et $w_{4}=1 / c_{0}$, avec $c_{0}=\sqrt{E / \rho}$ célérité des ondes à très basse fréquence. Le résultat est excellent. L'erreur 
maximale est inférieure à $4 \%$ pour un temps de mesure correspondant à 30 aller-retour des ondes dans la barre (figure 8). La durée utile de l'essai est donc beaucoup plus importante que celle d'une barre classique où un seul aller-retour est considéré. Le déplacement total au bout libre de la barre est ici de $8,9 \mathrm{~cm}$ ( 30 fois supérieur au déplacement qui serait mesuré par la méthode SHPB classique).

\section{Conclusion}

Nous avons présenté dans cet article une nouvelle méthode de séparation des ondes dans les barres élastiques et viscoélastiques utilisant plusieurs mesures de déformation et (ou) de vitesse. Une application expérimentale a été réalisée sur une barre d'aluminium. Elle montre que l'utilisation d'au moins trois mesures de déformation est nécessaire pour stabiliser le bruit retrouvé sur les valeurs des contraintes (déformations) calculées et que l'introduction d'une mesure de vitesse est indispensable pour stabiliser le calcul des déplacements (vitesses) en éliminant la dérive induite par la mesure imprécise des déformations nulles. L'utilisation de 3 mesures de déformation et d'une mesure de vitesse permet d'augmenter considérablement la durée totale de l'essai. Il est donc désormais possible de réaliser des mesures de qualité, sans limitation de la déformation maximum mesurable. Ceci donne accès à un domaine mal exploré situé entre celui que permettent d'atteindre les machines traditionnelles et celui auquel on accède avec les barres de Hopkinson.

\section{Références bibliographiques}

[1] Kolsky H., An investigation of the mechanical properties of materials at very high rates of loading, Proc. Phys. Soc. B 62 (1949) 676-700.

[2] Hopkinson B., A method of measuring the pressure in the detonation of high explosives or by an impact of bullets, Philos. Trans. Roy. Soc. Ser. A 231 (1914) 437-452.

[3] Campbell J.D., Duby J., The yield behaviour of mild steel in dynamic compression, Proc. Roy. Soc. London Ser. A 236 (1956) 24-40.

[4] Lundberg B., Henchoz A., Analysis of elastic waves in non-uniform rods from two-point strain measurement, Experiment. Mech. 17 (1977) 213-218.

[5] Park S.W., Zhou M., Separation of elastic waves in split Hopkinson bars using one-point strain measurements, Experiment. Mech. 39 (1999) 287-294.

[6] Zhao H., Gary G., Une nouvelle méthode de séparation des ondes pour l'analyse des essais dynamiques, C. R. Acad. Sci. Série II 319 (1994) 987-992.

[7] Zhao H., Gary G., A new method for the separation of waves. Application to the SHPB technique for an unlimited measuring duration, J. Mech. Phys. Solids 45 (1997) 1185-1202.

[8] Bacon C., Separating waves propagating in an elastic Hopkinson pressure bar with three-dimensional effects, Int. J. Impact Engng. 22 (1999) 55-69.

[9] Davies R.M., A critical study of Hopkinson pressure bar, Phil. Trans. Roy. Soc. Ser. A 240 (1948) 375-457.

[10] Lundberg B., Blanc R.H., Determination of mechanical material properties from the two-point response of an impacted linearly viscoelastic rod specimen, J. Sound Vibration 137 (1988) 483-493.

[11] Bussac M.N., Collet P., Gary G., Othman R., An optimisation method for separating and rebuilding onedimensional dispersive waves from multi-point measurements. Application to elastic or viscoelastic bars, J. Mech. Phys. Solids, 2001, in print.

[12] Pochhammer L., Uber die Fortpflanzungsgeschwindigkeinten kleiner Schwingungen in einem unbergrenzten isotropen Kreiszylinder, J. die Reine Angew. Math. 81 (1876) 324-336.

[13] Chree C., The equations of an isotropic elastic solid in polar and cylindrical co-ords, their solutions and applications, Cambridge Phil. Soc. Trans. 14 (1889) 250-369.

[14] Zhao H., Gary G., A three dimensional analytical solution of longitudinal wave propagation in an infinite linear viscoelastic cylindrical bar. Application to experimental techniques, J. Mech. Phys. Solids 43 (8) (1995) 13351348.

[15] Van Trees H.L., Detection, Estimation and Modulation Theory, Vol. I, Wiley, New York, 1968; Vol. III, Krieger, Malabar, 1992.

[16] Morse P.M., Feshbach H., Methods of Theoretical Physics, McGraw-Hill, New York, 1953.

[17] Hillström L., Mossberg M., Lundberg B., Identification of complex modulus from measured strains on an axially impacted bar using least squares, J. Sound Vibration 230 (3) (2000) 689-707. 